\title{
Avaliação dos fatores associados ao nível de estresse ocupacional das equipes de enfermagem que atuam nos serviços de urgência $e$ emergência
}

\author{
Evaluation of factors associated with the level of occupational stress of nursing teams that \\ work in the emergency and emergency services
}
Evaluación de los factores asociados con el nivel de estrés laboral de los equipos de enfermería que trabajan en servicios de urgencia y emergência

Vanessa de Souza Correia de Araujo ${ }^{1}$, Rhuana Maria de Oliveira Pereira ${ }^{2}$, Luana Oliveira de Souza ${ }^{1}$, Lucas da Silva de Almeida ${ }^{2 *}$, Priscilla Mendes Cordeiro², Sandra Jakeline Lima da Silva1, Marcelo Henrique da Silva Reis ${ }^{2}$, Jéssica Karoline Alves Portugal2 ${ }^{2}$, Ramanda Sena Guimarães², Tanny Thaylle Gomes de Souza².

\section{RESUMO}

Objetivo: Identificar e revisar os principais fatores associados ao nível de estresse ocupacional da equipe de enfermagem que atua em serviços de urgência e emergência. Métodos: Trata-se de uma revisão integrativa da literatura com busca por artigos científicos nas bases de dados Scientific Electronic Library Online (SCIELO) e Literatura Latino-Americana em Ciências da Saúde (LILACS) e Literatura Internacional em Ciências da Saúde (MEDLINE) de janeiro de 2009 a dezembro de 2019. Resultados: Os artigos selecionados versavam sobre os principais fatores desencadeantes do estresse ocupacional dos profissionais dos serviços de urgência e emergência, ambiente de trabalho, jornada de trabalho, situações de conflito vivenciados pelos profissionais, recursos humanos $(\mathrm{RH})$, equipamentos, materiais e superlotação. Considerações Finais: A enfermagem visa assistir o ser humano em todas as suas necessidades, exigindo do profissional habilidades cognitivas e comportamentais, sendo assim, é importante adotar ações preventivas e medidas práticas, a fim de reduzir os impactos sobre a saúde dos profissionais e o restabelecimento saúde física e mental dos trabalhadores.

Palavras-chave: Estresse ocupacional, Enfermagem, Urgência, Emergência.

\begin{abstract}
Objective: To identify and review the main factors associated with the level of occupational stress of the nursing team that works in urgent and emergency services. Methods: This is an integrative literature review with search for scientific articles in the Scientific Electronic Library Online (SCIELO) and Latin American Literature in Health Sciences (LILACS) and International Health Sciences Literature (MEDLINE) databases of January 2009 to December 2019. Results: The selected articles dealt with the main factors triggering occupational stress of professionals in urgent and emergency services, work environment, working hours, conflict situations experienced by professionals, human resources (HR), equipment, materials and overcrowding. Final Considerations: Nursing aims to assist the human being in all their needs, demanding from the professional cognitive and behavioral skills, therefore, it is important to adopt preventive actions and practical measures in order to reduce the impacts on the health of the professionals and the recovery physical and mental health of workers.
\end{abstract}

Keywords: Occupational stress, Nursing, Urgency, Emergency.

\footnotetext{
${ }^{1}$ Centro Universitário do Norte (UNINORTE), Manaus - AM.

2 Universidade Federal do Amazonas (UFAM), Coari - AM.

*E-mail: lucas.enf.almeida@hotmail.com
} 


\section{RESUMEN}

Objetivo: Identifique y revise los principales factores asociados con el nivel de estrés ocupacional del equipo de enfermería que trabaja en los servicios de urgencia y emergencia. Métodos: Esta es una revisión bibliográfica integradora con búsqueda de artículos científicos en las bases de datos de la Biblioteca electrónica científica en línea (SCIELO) y Literatura latinoamericana en ciencias de la salud (LILACS) y Literatura internacional en ciencias de la salud (MEDLINE) de enero de 2009 a diciembre de 2019. Resultados: los artículos seleccionados abordaron los principales factores que desencadenan el estrés laboral de los profesionales en servicios de urgencia y emergencia, ambiente de trabajo, horas de trabajo, situaciones de conflicto experimentadas por profesionales, recursos humanos (HR), equipos, materiales y hacinamiento. Consideraciones finales: la enfermería tiene como objetivo ayudar a los seres humanos en todas sus necesidades, exigiendo habilidades cognitivas y conductuales profesionales, por lo tanto, es importante adoptar acciones preventivas y medidas prácticas, a fin de reducir los impactos en la salud de los profesionales y la recuperación salud física y mental de los trabajadores.

Palabras clave: Estrés laboral, Enfermería, Urgencia, Emergencia.

\section{INTRODUÇÃO}

O estresse e estressores podem ser compreendidos como toda e qualquer causa interna ou social, que atua no psiquismo do indivíduo, ativando emoções, dentre elas, destacamos: o medo, a raiva, a ambição, a culpa, dentre outros, que são fatores capazes de provocar uma reação do sistema nervoso glandular, e consequentemente, acarretando consequências, principalmente, ao nível físico, onde cada indivíduo possui uma quantidade exclusiva de energia adaptativa, na qual é limitada (SILVA RS, et al., 2010).

Quando recebe um estímulo, o organismo desencadeia uma resposta, como o preparo para fugir ou reagir do confronto da situação, de acordo com a vulnerabilidade individual e integrando a esfera físico-psicossocial, leva a transformações orgânicas e mentais, de forma extensa e diversificada (SILVA RS, et al., 2010).

Nesse sentido, considera-se o estresse relacionado ao trabalho como uma soma de perturbações psicológicas ou sofrimento psíquico, estando associado às experiências de trabalho, provocando o estresse ocupacional, em que a pessoa identifica este como ameaçador às suas necessidades de realização pessoal e profissional, como também à sua saúde física e mental, de certa forma, afetando a interação desta com o trabalho e com o seu ambiente, gerando sintomas como: pressa, conflitos interpessoais, desmotivação, agressividade, entre outros (SILVA RS, et al., 2010).

As principais fontes de estresse podem ser advindas de fatores ambientais, como por exemplo: ruído, iluminação, temperatura e ventilação em limites inadequados, como também de fatores organizacionais, além do envolvimento e participações no trabalho, suportes organizacionais, organização do trabalho, sendo este com apoio nos conceitos mentais, monotonia ou sobrecarga de trabalho, ritmo de produção e de trabalho, pressões temporais e o significado do trabalho, da natureza e das tarefas (SILVA RS, et al., 2010).

A área de urgência e emergência hospitalar é um setor que exige dos profissionais de enfermagem um conhecimento aprofundado em relação às diversas situações de saúde e esta precisa ter controle sobre as especificidades da assistência, como por exemplo, o raciocínio ágil, habilidade manual e resolutividade das adversidades que se manifestam no ambiente de trabalho, além de ser flexível, tendo em vista a grande quantidade de procedimentos a serem realizados, o estado clínico do paciente e a limitação do período de tempo (COSTA AF, et al., 2005).

No cenário de urgência e emergência hospitalar, os profissionais de enfermagem lidam diariamente com uma grande demanda de pacientes com risco iminente de morte, eventualidades de natureza imprevisíveis, amplas jornadas de trabalho, pressão de chefia, exigência de familiares e tempo reduzido para prestação de assistência, em que a complexidade dos cuidados prestados, somada a fatores pessoais, tem relação constante com desencadeamento do estresse (BEZERRA FN, et al., 2012).

Portanto, é de suma importância que o enfermeiro que atua no cenário de urgência e emergência reconheça os estressores em seu ambiente de trabalho e suas consequências no processo saúde-doença, 
além disto, procure soluções para atenuá-los e confrontá-los, evitando danos à sua saúde e ofertando uma assistência qualificada aos usuários, mas para isso ocorrer, necessita deixar as diferenças de lado e trabalhar em equipe com demais profissionais e também com gestores (BEZERRA FN, et al., 2012).

A partir dessas informações, questiona-se: Quais os principais fatores desencadeantes do estresse ocupacional dos profissionais de enfermagem que exercem sua assistência nos serviços de urgência e emergência? Diante disto, o presente estudo tem como objetivo identificar, através de uma revisão integrativa da literatura, os principais fatores associados ao nível de estresse ocupacional da equipe de enfermagem que atua em serviços hospitalares de urgência e emergência.

\section{MÉTODOS}

Trata-se de um estudo de Revisão Integrativa da Literatura (RIL), realizado a partir de pesquisas em artigos científicos publicados nas bases de dados Scientific Electronic Library Online (SCIELO), Literatura LatinoAmericana em Ciências da Saúde (LILACS), Literatura Internacional em Ciências da Saúde (MEDLINE) e Base de dados em Enfermagem (BDENF), utilizando os seguintes descritores: enfermagem, estresse ocupacional, urgência e emergência.

A revisão integrativa da literatura é um método que determina o conhecimento específico sobre uma temática, visando identificar, analisar e sintetizar resultados de estudos independentes que retratam o mesmo assunto, para uma possível repercussão favorável na qualidade dos cuidados prestados ao paciente.

Como critérios de inclusão foram estabelecidos artigos em português, publicados de janeiro de 2009 a dezembro de 2019 e que correspondessem aos objetivos da revisão. Os critérios de exclusão foram: estudos do tipo teses, dissertações, monografias e revisão de literatura.

Por tratar-se de um estudo de revisão da literatura, não foi necessária a submissão do trabalho no Comitê de Ética em Pesquisa (CEP), no entanto, a elaboração foi realizada respeitando as normas de citação e preservação dos direitos autorais vigentes.

\section{RESULTADOS}

Para elaboração desta revisão de literatura foram identificados na base de dados um total de 108 artigos. $\mathrm{Na}$ base SCIELO foram encontradas inicialmente 3 referências, após a aplicabilidade dos filtros baseados nos critérios de inclusão e exclusão e leitura minuciosa, foram selecionados 2 artigos para compor a amostra. $\mathrm{Na}$ base de dados LILACS, foram obtidas 28 publicações, após a filtragem utilizando os critérios de inclusão e exclusão, restaram 11 artigos, após a leitura minuciosa restaram 8 artigos para compor a amostra.

$\mathrm{Na}$ base de dados MEDLINE foram obtidas 42 publicações, após a filtragem utilizando os critérios de inclusão e exclusão, o resultado de pesquisa foi 0 , não havendo publicações para compor a amostra. Na base de dados BDENF foram levantadas 35 publicações, após a aplicabilidade dos filtros baseados no critério de inclusão e exclusão e leitura minuciosa, foram selecionados 2 artigos para a composição da amostra. Assim, a amostra final utilizada para compor esta pesquisa foi de 12 artigos, conforme exposto na Figura 1. 
Figura 1 - Fluxograma metodológico para seleção de artigos.

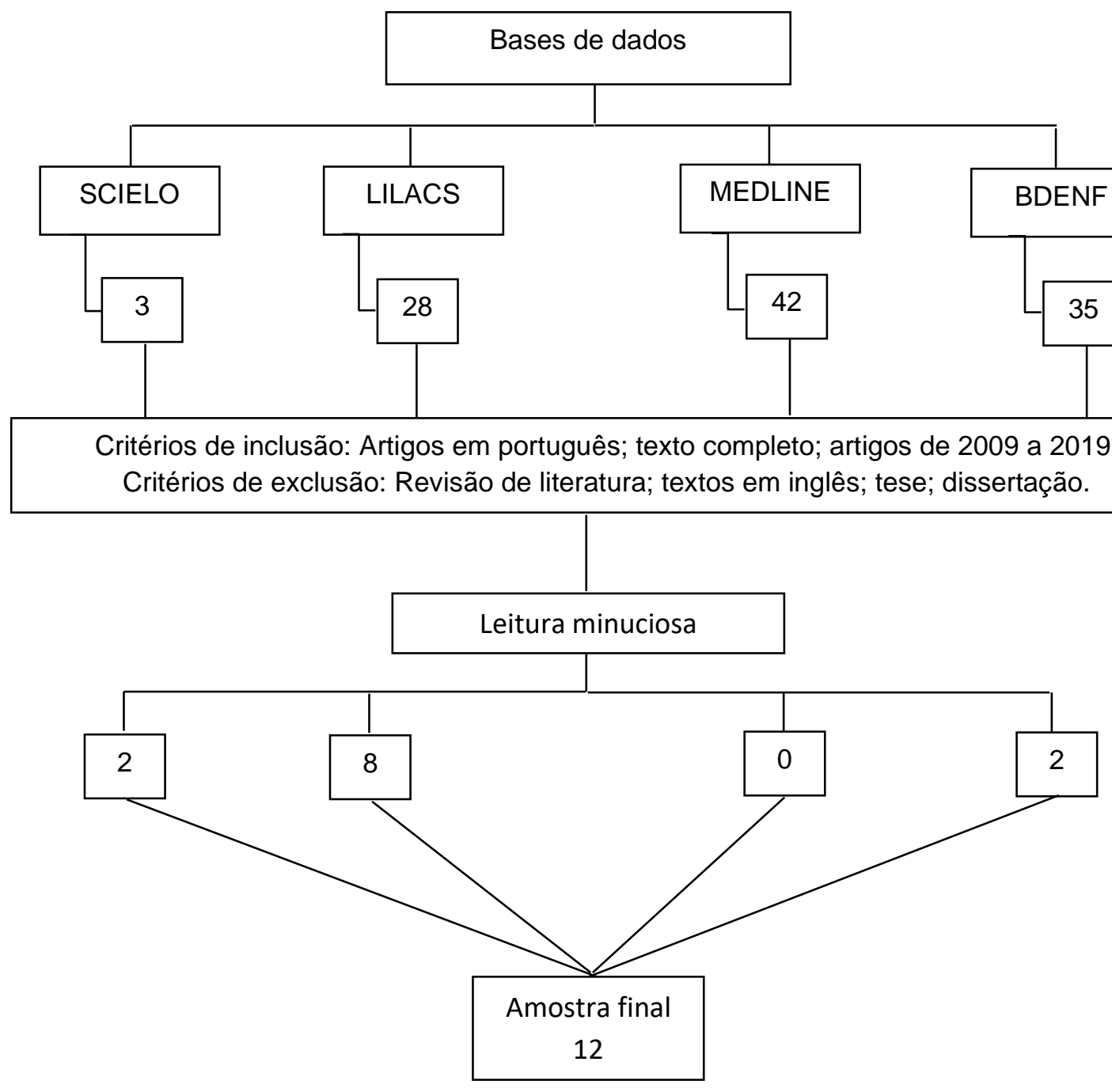

Fonte: Araujo VSC, et al., 2019. 
Quadro 1 - Sumário dos estudos e seus principais resultados para avaliação dos fatores associados ao nível de estresse ocupacional das equipes de enfermagem que atuam nos serviços de urgência e emergência, $n=12$. Manaus - AM.

\begin{tabular}{|c|c|c|c|c|c|c|c|c|}
\hline № & Base & Revista & Título & Autor(es) & Objetivo & \multicolumn{2}{|c|}{ Metodologia } & Ano \\
\hline \multirow[b]{2}{*}{1} & \multirow[b]{2}{*}{ LILACS } & \multirow[b]{2}{*}{$\begin{array}{l}\text { Journal Health } \\
\text { NPEPS }\end{array}$} & \multirow{2}{*}{$\begin{array}{c}\text { Autopercepção do } \\
\text { estresse ocupacional na } \\
\text { equipe de enfermagem } \\
\text { de um serviço de } \\
\text { emergência }\end{array}$} & \multirow[b]{2}{*}{$\begin{array}{l}\text { Silva PN, et } \\
\text { al. }\end{array}$} & \multirow{2}{*}{$\begin{array}{l}\text { Identificar o perfil e a } \\
\text { autopercepção do } \\
\text { estresse na equipe de } \\
\text { enfermagem atuante } \\
\text { em setor de emergência }\end{array}$} & Tipo de estudo & Abordagem & \multirow[b]{2}{*}{2019} \\
\hline & & & & & & $\begin{array}{c}\text { Transversal/Descritiva/ } \\
\text { Exploratório }\end{array}$ & Quantitativo & \\
\hline 2 & LILACS & $\begin{array}{l}\text { Rev enferm } \\
\text { UERJ }\end{array}$ & $\begin{array}{c}\text { Equipe de enfermagem } \\
\text { de emergência: riscos } \\
\text { ocupacionais e medidas } \\
\text { de autoproteção }\end{array}$ & $\begin{array}{l}\text { Martins JT, } \\
\text { et al. }\end{array}$ & $\begin{array}{c}\text { Desvelar o } \\
\text { conhecimento de risco } \\
\text { no trabalho e identificar } \\
\text { as medidas de } \\
\text { autoproteção utilizadas } \\
\text { por uma equipe de } \\
\text { enfermagem de pronto } \\
\text { socorro de um hospital } \\
\text { universitário de } \\
\text { Londrina, Paraná. }\end{array}$ & Descritiva & Qualitativa & 2014 \\
\hline 3 & LILACS & $\begin{array}{c}\text { Rev Bras Med } \\
\text { Trab }\end{array}$ & $\begin{array}{l}\text { Estresse ocupacional } \\
\text { dos enfermeiros de } \\
\text { urgência e emergência } \\
\text { de um hospital público } \\
\text { de Teresina (PI) }\end{array}$ & $\begin{array}{l}\text { Santana } \\
\text { RS, et al. }\end{array}$ & $\begin{array}{l}\text { Verificar o nível de } \\
\text { estresse ocupacional } \\
\text { dos enfermeiros pela } \\
\text { Escala Bianchi na } \\
\text { unidade de urgência e } \\
\text { emergência de um } \\
\text { hospital público de } \\
\text { Teresina, Piauí. }\end{array}$ & Transversal/Descritivo & Quantitativo & 2019 \\
\hline 4 & LILACS & $\begin{array}{l}\text { Rev enferm } \\
\text { UERJ }\end{array}$ & $\begin{array}{c}\text { Estresse ocupacional e } \\
\text { burnout em enfermeiros } \\
\text { de um serviço de } \\
\text { emergência: a } \\
\text { organização do trabalho }\end{array}$ & $\begin{array}{l}\text { Oliveira EB, } \\
\text { et al. }\end{array}$ & $\begin{array}{l}\text { Analisar as dimensões } \\
\text { da Síndrome de } \\
\text { Burnout em enfermeiros } \\
\text { que trabalham em } \\
\text { serviço de emergência. }\end{array}$ & Transversal/Descritivo & Quantitativo & 2017 \\
\hline
\end{tabular}

REAS/EJCH | Vol.Sup.n.52 | e3586 | DOI: https://doi.org/10.25248/reas.e3586.2020 Página 5 de 11 
Revista Eletrônica Acervo Saúde / Electronic Journal Collection Health | ISSN 2178-2091

\begin{tabular}{|c|c|c|c|c|c|c|c|c|}
\hline 5 & LILACS & Rev Min Enferm & $\begin{array}{l}\text { Estresse ocupacional no } \\
\text { serviço de atendimento } \\
\text { móvel de urgência }\end{array}$ & $\begin{array}{c}\text { Andrade } \\
\text { MCM e } \\
\text { Junior ACS. }\end{array}$ & $\begin{array}{c}\text { Avaliar os níveis de } \\
\text { estresse ocupacional na } \\
\text { equipe do Serviço de } \\
\text { Atendimento Móvel de } \\
\text { Urgência (SAMU) da } \\
\text { cidade de } \\
\text { Marília }\end{array}$ & Descritiva & Investigatória & 2014 \\
\hline 6 & LILACS & Rev. Eletr. Enf & $\begin{array}{c}\text { Estressores e coping: } \\
\text { enfermeiros de uma } \\
\text { unidade de emergência } \\
\text { hospitalar }\end{array}$ & $\begin{array}{l}\text { Silveira } \\
\text { MM, et al. }\end{array}$ & $\begin{array}{l}\text { Identificar estressores } \\
\text { vivenciados por } \\
\text { enfermeiros que atuam } \\
\text { em uma Unidade de } \\
\text { Emergência de um } \\
\text { hospital geral, } \\
\text { mecanismos de coping } \\
\text { para lidar com o } \\
\text { estresse no trabalho, } \\
\text { bem como as } \\
\text { repercussões na } \\
\text { assistência ao usuário. }\end{array}$ & Transversal/ Analítica & Quantitativa & 2009 \\
\hline 7 & LILACS & $\begin{array}{l}\text { Acta Paul } \\
\text { Enferm }\end{array}$ & $\begin{array}{c}\text { Preditores da Síndrome } \\
\text { de Burnout em } \\
\text { enfermeiros de serviços } \\
\text { de urgência pré- } \\
\text { hospitalar }\end{array}$ & $\begin{array}{c}\text { França } \\
\text { SPS, et al. }\end{array}$ & $\begin{array}{l}\text { Analisar os preditores } \\
\text { da Síndrome de } \\
\text { Burnout apresentados } \\
\text { por enfermeiros de } \\
\text { serviços de urgência } \\
\text { pré-hospitalar móvel. }\end{array}$ & $\begin{array}{l}\text { Descritivo/ } \\
\text { Exploratório }\end{array}$ & Quantitativo & 2012 \\
\hline 8 & LILACS & $\begin{array}{l}\text { Acta Paul } \\
\text { Enferm }\end{array}$ & $\begin{array}{l}\text { Sintomas de depressão } \\
\text { e fatores intervenientes } \\
\text { entre enfermeiros de } \\
\text { serviço hospitalar de } \\
\text { emergência }\end{array}$ & $\begin{array}{c}\text { Oliveira FP, } \\
\text { et al. }\end{array}$ & $\begin{array}{c}\text { Verificar se enfermeiros } \\
\text { do serviço hospitalar de } \\
\text { emergência } \\
\text { presentavam sintomas } \\
\text { depressivos, identificar } \\
\text { fatores intervenientes e } \\
\text { analisar percepção } \\
\text { sobre o sofrimento } \\
\text { psíquico e influência na } \\
\text { assistência prestada. }\end{array}$ & Transversal & Quantitativo & 2015 \\
\hline
\end{tabular}

REAS/EJCH | Vol.Sup.n.52 | e3586 | DOI: https://doi.org/10.25248/reas.e3586.2020 Página 6 de 11 
Revista Eletrônica Acervo Saúde / Electronic Journal Collection Health | ISSN 2178-2091

\begin{tabular}{|c|c|c|c|c|c|c|c|c|}
\hline 9 & BDENF & $\begin{array}{c}\text { Revista Online } \\
\text { de Pesquisa }\end{array}$ & $\begin{array}{c}\text { A enfermagem na } \\
\text { urgência e emergência: } \\
\text { entre o prazer e o } \\
\text { sofrimento }\end{array}$ & $\begin{array}{l}\text { Kolhs M, et } \\
\text { al. }\end{array}$ & $\begin{array}{c}\text { Verificar quais os } \\
\text { fatores que levam } \\
\text { prazer e sofrimento aos } \\
\text { profissionais da } \\
\text { enfermagem que atuam } \\
\text { em um setor de } \\
\text { urgência e emergência } \\
\text { hospitalar, e estratégias } \\
\text { defensivas. } \\
\end{array}$ & Descritivo & Qualitativo & 2017 \\
\hline 10 & BDENF & $\begin{array}{l}\text { Revista de } \\
\text { pesquisa: } \\
\text { Cuidado é } \\
\text { Fundamental }\end{array}$ & $\begin{array}{c}\text { O desgaste relacionado } \\
\text { ao trabalho na ótica dos } \\
\text { enfermeiros de } \\
\text { atendimento pré- } \\
\text { hospitalar }\end{array}$ & $\begin{array}{l}\text { Martins } \\
\text { CCF, et al. }\end{array}$ & $\begin{array}{c}\text { Identificar como } \\
\text { enfermeiros do Serviço } \\
\text { de Atendimento Móvel } \\
\text { às Urgências (SAMU) } \\
\text { percebem o } \\
\text { desgaste relacionado } \\
\text { ao trabalho cotidiano. }\end{array}$ & Descritivo & Qualitativa & 2011 \\
\hline 11 & SCIELO & $\begin{array}{l}\text { Revista } \\
\text { Brasileira de } \\
\text { Enfermagem }\end{array}$ & $\begin{array}{c}\text { Sentimentos } \\
\text { vivenciados pelos } \\
\text { profissionais de } \\
\text { enfermagem que atuam } \\
\text { em unidade de } \\
\text { emergência }\end{array}$ & $\begin{array}{l}\text { Salomé } \\
\text { GM, et al. }\end{array}$ & $\begin{array}{l}\text { Conhecer o significado } \\
\text { do trabalho em uma } \\
\text { unidade de emergência } \\
\text { para os profissionais de } \\
\text { enfermagem. }\end{array}$ & Qualitativa & Fenomenológica & 2009 \\
\hline 12 & SCIELO & $\begin{array}{l}\text { Acta Paul } \\
\text { Enferm }\end{array}$ & $\begin{array}{l}\text { Síndrome de Burnout } \\
\text { em trabalhadores de } \\
\text { enfermagem de um } \\
\text { pronto socorro de } \\
\text { hospital universitário }\end{array}$ & $\begin{array}{c}\text { Jodas DA e } \\
\text { Haddad } \\
\text { MC. }\end{array}$ & $\begin{array}{l}\text { Investigar os sinais e } \\
\text { sintomas de burnout em } \\
\text { trabalhadores de } \\
\text { enfermagem de um } \\
\text { Pronto Socorro de } \\
\text { Hospital Universitário, } \\
\text { correlacionando-os com } \\
\text { fatores preditores. }\end{array}$ & Descritivo & Quantitativo & 2009 \\
\hline
\end{tabular}

Fonte: Araujo VSC, et al., 2019. 


\section{DISCUSSÃO}

Após a análise dos artigos selecionados para constituir a amostra da pesquisa, foram utilizados blocos de conteúdo temáticos que conduziram os achados frequentes, como forma de descrever quais os principais fatores desencadeantes do estresse ocupacional dos profissionais de enfermagem que exercem sua assistência nos serviços de urgência e emergência.

\section{O local e a jornada de trabalho nas urgências e emergências como principal fator estressor.}

Martins JT, et al. (2011) (Artigo 10), as condições do ambiente de trabalho influenciam na saúde do trabalhador, podendo comprometer sua saúde mental e o seu desempenho profissional. Segundo Silva PN, et al. (2019) (Artigo 1), a jornada de trabalho no setor de urgência e emergência gera uma somatória de estressores, sendo eles ambientais (ruído, iluminação, temperatura, ventilação inapropriada) e organizacionais (tarefas acima da capacidade diária e a pressão pelo atendimento ágil e eficaz) que contribuem de forma significativa no desenvolvimento dos quadros de estresse na equipe.

A maioria dos profissionais de enfermagem entrevistados que atuam no Serviço de Emergência em Barra do Garças responsabiliza o ambiente de trabalho vivenciado nas unidades de urgência como o maior causador do quadro de estresse, devido a rotina de trabalho, a alta demanda de pacientes que procuram atendimento, as situações de conflito e o desgaste físico e mental, onde passaram a manifestar alterações cognitivas, físicas, emocionais e comportamentais em maior ou menor grau (SILVA PN, et al., 2019).

De acordo com Fonseca JRF e Neto DL (2014), no ambiente de trabalho em emergência, o enfermeiro vivência situações desafiadoras, como por exemplo: elevada carga de trabalho e complexidade das atividades assistenciais e gerenciais concomitantes, as quais são percebidas como desgastantes e geradoras de respostas negativas de estresse, e por seguinte comprometedoras das quatros áreas: social, familiar/afetivo, saúde e profissional.

Neste contexto, a junção de fatores principalmente ligados a árdua jornada de trabalho e escassez de recursos e materiais afetam negativamente na saúde destes trabalhadores.

No pesquisa realizada por Filho AM e Araújo TM (2015), os profissionais participantes relatam que a escassez quanto ao ambiente de trabalho, a precariedade dos equipamentos técnicos $(51,1 \%)$, falta de equipamento de proteção individual $(48,9 \%)$ e insuficiência nos recursos gerais $(71,3 \%)$ são os fatores que mais afetam na hora de prestar a assistência, sobrecarregando o profissional, dificultando a prestação de serviços da boa qualidade e gerando estressores no ambiente de trabalho, tornando-o propício para o desenvolvimento de problemas psicológicos.

Santos JNMO, et al. (2019), aborda em seu estudo o estresse ocupacional da equipe de enfermagem, em entrevistas conduzidas no fim do expediente dos profissionais. Dentre os principais fatores relatados, destacam-se: o cansaço físico por estar em uma rotina de idas e vindas durante os procedimentos, pressão por conduzir grandes responsabilidades, dores na coluna, cefaleia e o desgaste emocional.

Silva PN, et al. (2019) (Artigo 3), explana em seu estudo que o elevado nível de estresse ao qual os profissionais de saúde estão sujeitos, ocorre devido os frequentes obstáculos vivenciados principalmente pelos relacionamentos com outras unidades e supervisores na rotina diária de trabalho, expondo esses profissionais à situações de sofrimento, morte, extensas jornadas de trabalho e exigências institucionais.

O trabalho exercido pelos enfermeiros é uma atividade diferenciada de grande vulnerabilidade ao estresse, pois os mesmos realizam a assistência diretamente com o paciente e são responsáveis pela gerência do cuidado. Em função dessas atribuições, o enfermeiro assume posição de líder na equipe de saúde, o que lhe propicia desenvolver métodos que intensificam o trabalho em equipe e a organização do ambiente assistencial, visando assim a qualidade do cuidado prestado (SILVA PN, et al., 2019).

Segundo Silva PN, et al. (2019), os profissionais que prestam uma atividade por tempo prolongado em determinado setor, acarretam elevado nível de estresse, ocorrendo principalmente pela trivialização da dinâmica de trabalho. Por outro lado, baseado em análise das variáveis de trabalho, a exaustão é maior naqueles profissionais com menos período de instituição, expondo que o descontentamento com o trabalho e o reduzido apoio social diz respeito ao elevado desgaste laboral.

REAS/EJCH | Vol.Sup.n.52 | e3586 | DOI: https://doi.org/10.25248/reas.e3586.2020 Página 8 de $1 \mathbf{1}$ 
Oliveira EB, et al. (2017) (Artigo 4), mencionam que o setor de emergência, é o ambiente de trabalho onde há maior ocorrência de licenças na enfermagem por problemas de saúde, por se tratar de um ambiente ocupacional identificado por eventos de estresse, devido ao convívio com a dor, o sofrimento, a morte e os acidentes, marcado por quadro escasso de trabalhadores, a ambiguidade de papéis, na qual o enfermeiro precisa se desmembrar para atender as incontáveis demandas de cunho técnico e gerencial, como também as longas jornadas de trabalho semanais, sendo maior de 44 horas, se sujeitando a diversas cargas de trabalho causadoras de processos de desgaste e adoecimento, afetando a saúde e a qualidade da assistência.

Oliveira FP, et al. (2015) (artigo 8) e Kolhs M, et al. (2017) (Artigo 9), corroboram com a mesma ideia de que o trabalho pode ser responsável por estabelecer-se motivação de prazer ou de sofrimento, dependendo do modo que é organizado, pois os profissionais não lidam apenas com o corpo dos pacientes, mas também com o espiritual num todo.

A dinâmica do trabalho tem foco nas ações empregadas para verificar a organização do trabalho utilizada pelos profissionais. A partir disto, são produzidas estratégias que fornecem ou não a possibilidade para 0 desenvolvimento do sofrimento, prazer, reconhecimento, cooperação, o que pode propiciar a saúde ou o adoecimento (OLIVEIRA FP, et al., 2015; KOLHS M, et al., 2017).

De acordo com a pesquisa dos autores Oliveira FP, et al. (2015) e Kolhs M, et al. (2017), os enfermeiros informaram como causadores pela evolução de seu quadro depressivo a sobrecarga de trabalho, o baixo salário, carga horária excessiva, desgaste e preocupação com o trabalho. Essas questões estão vinculadas a maneira como o mercado de trabalho tem se demonstrado, devido à alta demanda de profissionais para um pequeno número de vagas de emprego, levando assim ao desemprego e formação de exército de reserva, que por sua vez ocasiona a desvalorização da profissão.

Por conseguinte, isso acaba gerando cargas elevadas de trabalho e o nível físico e psíquico dos indivíduos resulta em cansaço físico e estresse para o trabalhador.

Salomé GM, et al. (2009) (Artigo 11), aborda que situações como: a insuficiência de recursos humanos para a prestação da assistência rotineira e emergencial aos pacientes, acarretam o sofrimento mental e físico do profissional. Assim, compete-lhes tomar decisões críticas que incitam demasiada carga afetiva, uma vez que lidam rotineiramente com a angústia dos pacientes e familiares. Cabe-lhes ainda oferecer suporte ao paciente e família, além de realizar adaptações radicais no processo de trabalho, quase sempre sob circunstâncias extremamente precárias.

Seguindo a mesma linha de pensamento, o estudo realizado por Fonseca JRF e Neto DL (2014), relata que atender aos familiares dos pacientes críticos foi considerada a atividade com maior estresse, seguido por orientar familiares de pacientes e atender as necessidades das famílias. Santos JNMO, et al. (2019), enfatiza que a pressão e sobrecarga causadas por parte dos acompanhantes se torna mais um fator a que equipe de enfermagem está exposta e que pode levá-la ao cansaço e estresse, acometendo sua saúde mental.

Além da pressão e sobrecarga, a não valorização do trabalho de enfermagem por parte da sociedade e principalmente na área hospitalar. Assim, a grande demanda de trabalho de recursos materiais e humanos fazem com o que o trabalho de enfermagem se torne exaustivo e estressante, dificultando assim as relações entre estes profissionais, acompanhantes e pacientes (SANTOS JNMO, et al., 2019). Salomé GM, et al. (2009) (Artigo 11), relata que os principais fatores de risco discutidos se relacionam a desorganização do trabalho e aos fatores ambientais e ergonômicos inapropriados. Dentre eles, o deslocamento e o transporte de pacientes, a postura corporal indevida, o déficit de profissional e os equipamentos inadequados e sem manutenção.

\section{Os riscos ocupacionais como um determinante agente estressante.}

Segundo Silva NP, et Al. (2019) (Artigo 1), a jornada de trabalho no âmbito da urgência e emergência constitui uma soma de estressores desencadeados pelo ambiente e a organização na qual o profissional se situa. O mesmo autor relata ainda que alguns fatores como: o ruído, a iluminação, temperatura, falta de ventilação apropriada, fatores ambientais, encargos diários, alta demanda de pacientes e a pressão pela assistência ágil e eficaz, favorecem no adiantamento dos cenários de esgotamento da equipe de urgência e

REAS/EJCH | Vol.Sup.n.52 | e3586 | DOI: https://doi.org/10.25248/reas.e3586.2020 Página 9 de 11 
emergência. Outros fatores determinantes vivenciados pelos profissionais desta categoria são episódios de conflitos, desgaste físico, mental, nervosismo e ansiedade, que vão desde a intensa rotina de trabalho, condições trabalhistas, baixa remuneração, fatores organizacionais, que influenciam arduamente na saúde física e mental desses profissionais.

Martins JT, et al. (2019) (Artigo 2), divide em subcategorias os principais fatores que predispõem os riscos ocupacionais em que os profissionais de urgência e emergência estão passíveis, na qual relaciona-se diretamente na assistência aos usuários deste serviço, especialmente em um eventual contato com fluídos, sangue, dispositivos contaminados que expõe os mesmos a infecções e doenças desconhecidas, procedimentos e intervenções que carecem de materiais perfurocortantes, além de pacientes dependentes que necessitam de auxílio dos trabalhadores requerendo seus esforços físicos. Os recursos humanos e equipamentos são outras subcategorias que recebem destaque por Martins JT, et al. (2019), segundo os autores, elas apresentam números insuficientes para que seja realizada a prestação de serviços de qualidade.

No estudo em questão, os entrevistados informaram que se encontram diante de elevados riscos devido à inadequação do $\mathrm{RH}$, visto que a falta de funcionários atrelada a deficiência de equipamentos primordiais é um dos grandes empecilhos, diante disto, ocorre uma insegurança para a realização das suas atividades laborais, ainda mais em um setor onde a demanda tende a ser relativamente grande. Menciona-se que é constante a superlotação nesses serviços de saúde, visto que o número de profissionais de enfermagem é escasso para atender a demanda, o que causa o excesso de atividades, aumenta o estresse e o desgaste dos mesmos, e consequentemente, decai a qualidade da assistência, podendo ocasionar um possível acidente de trabalho, bem como iatrogenia aos usuários que usufruem deste serviço, visto que, são feitos que podem implicar no progresso de distúrbios físicos e/ou mentais.

Dentre os distúrbios físicos e/ou mentais, os profissionais estão facilmente vulneráveis ao desenvolvimento da síndrome de Burnout. Trettene AS, et al. (2016), menciona em um estudo realizado com trabalhadores de um Pronto Socorro de um Hospital Universitário, onde $8 \%$ dos profissionais possuíam a síndrome e 54\% apresentavam alto risco para a manifestação da doença. Além disto, Trettene AS, et al. (2016), evidencia o desenvolvimento da síndrome por meio da associação entre o maior tempo de atuação do profissional, levando em consideração o turno vespertino devido à grande demanda de ocorrências de estresse e consequentemente, desencadeando maiores níveis exaustivos.

\section{Fatores externos que contribuem significativamente para a ocorrência da tensão ocupacional.}

Silveira MM, et al. (2009) (Artigo 6), em seu artigo agrega enigmas vivenciados no meio de trabalho com o ambiente familiar: para $26,3 \%$ dos profissionais enfermeiros muitas vezes ou sempre conciliar estas questões torna-se uma ação estressante, que afeta no meio laboral, para $31,6 \%$ destes profissionais apenas algumas vezes é estressante.

Grande parte desta população, o que predomina é a população do gênero feminino, que desenvolvem suas vidas como pessoas, esposas, mães, gerenciando simultaneamente com as suas atividades profissionais. Andrade MCM e Júnior ACS (2014) (Artigo 5), o casamento representa uma renúncia aos descompromissos da vida de solteiro, determinando a construção de novas responsabilidades, as mudanças do matrimônio resultam em amadurecimento e foco em relação aos compromissos profissionais.

Segundo Souza SPF, et al. (2012) (Artigo 7), o fato de ter ou não filhos, do mesmo modo a quantidade dos mesmos, é uma variável controversa para alguns pesquisadores, pois o fato de possuir filhos é visto como uma razão de equilíbrio para os profissionais, proporcionando excelentes métodos de enfrentamento de acontecimentos conflituosos.Jodas DA e Haddad MCL (2009) (Artigo 12) e Kolhs M, et al. (2017) (Artigo 9), referem que os sentimentos de impotência, desânimo, insatisfação, culpa, tristeza e outro que concerne emoções, resultam em desgaste físico e emocional, afetando o rendimento laboral e potencialmente levando o profissional ao adoecimento psíquico.

\section{CONSIDERAÇÕES FINAIS}

A partir deste estudo, foi possível constatar que os serviços de saúde no setor de urgência e emergência geram uma somatória de estressores, fatores que contribuem de modo significativo no desenvolvimento dos quadros de estresse e até mesmo de patologias psíquicas na equipe de saúde, onde os principais causadores

REAS/EJCH | Vol.Sup.n.52 | e3586 | DOI: https://doi.org/10.25248/reas.e3586.2020 Página 10 de $1 \mathbf{1}$ 
apontados nesta pesquisa foram: fatores ambientais, organizacionais, alta demanda de pacientes procurando atendimento, árdua jornada de trabalho, escassez de recursos materiais e outros.

Neste sentido, é importante destacar a necessidade da implementação de um programa de prevenção do estresse ocupacional, tendo em vista a adoção de ações a fim de reduzir os impactos sobre a saúde dos profissionais, além da implementação de medidas que estabeleça uma melhor qualidade de vida.

\section{REFERÊNCIAS}

1. ANDRADE MCM, JÚNIOR ACS. Estresse ocupacional no serviço de atendimento móvel de urgência. Revista Mineira de enfermagem. 2014; 18(2): 376-391.

2. BEZERRA FN, et al. Estresse ocupacional dos enfermeiros de urgência e emergência: Revisão Integrativa da Literatura. Acta Paulista de Enfermagem. 2012; 25(2): 151-156.

3. COSTA AF, et al. O trabalho do enfermeiro no setor de urgência/emergência hospitalar. IX Encontro Latino Americano de Pós-Graduação-Universidade do Vale do Paraíba, 2005.

4. FILHO AM, ARAÚJO TM. Estresse ocupacional e saúde mental dos profissionais do centro de especialidades médicas de Aracaju. Trab. Educ. Saúde, Rio de Janeiro. 2015; 13(supl.1): 177-199.

5. FONSECA JRF, NETO DL. Nível de estresse ocupacional e atividades estressoras em enfermeiros de unidades de emergência. Rev Rene. 2014; 15(5): 732-42.

6. JODAS DA, HADDAD MCL. Síndrome de Burnout em trabalhadores de enfermagem de um pronto socorro de hospital universitário. Acta paulista de enfermagem. 2009; 22(2): 192-197.

7. KOLHS M, et al. A enfermagem na urgência e emergência: entre o prazer e o sofrimento. Revista de Pesquisa: Cuidado é Fundamental Online. Rio de Janeiro. 2017; 9(2): 422-431.

8. MARTINS JT, et al. Equipe de enfermagem de emergência: riscos ocupacionais e medidas de autoproteção [Emergency nursing team: occupational risks and self protection]. Revista Enfermagem UERJ. 2014; 22(3): 334-340.

9. MARTINS CCF, et al. O desgaste relacionado ao trabalho na ótica dos enfermeiros de atendimento préhospitalar. Revista de Pesquisa Cuidado é Fundamental Online. 2011; 3(2): 2024-2032.

10. OLIVEIRA EB, et al. Estresse ocupacional e burnout em enfermeiros de um serviço de emergência: a organização do trabalho. Rev. enferm. UERJ. 2017; 25: [e28842]-[e28842].

11. OLIVEIRA FP, et al. Sintomas de depressão e fatores intervenientes entre enfermeiros de serviço hospitalar de emergência. Acta Paulista de Enfermagem. 2015; 28(3): 209-215.

12. SALOMÉ GM, et al. Sentimentos vivenciados pelos profissionais de enfermagem que atuam em unidade de emergência. Revista Brasileira de Enfermagem. 2009; 62(6): 856-862.

13. SANTOS JNMO, et al. Estresse ocupacional: exposição da equipe de enfermagem de uma unidade de emergência. J. res.: fundam. care. Online. 2019; 11 (n.esp): 455-463.

14. SILVA JFC. Estresse ocupacional e suas principais causas e consequências. Universidade Cândido Mendes. Instituto a vez do mestre, 2010.

15. SILVA PN, et al. Autopercepção do estresse ocupacional na equipe de enfermagem de um serviço de emergência/Self-perceived occupational stress in the nursing team of an emergency service/Estrés ocupacional autopercibido en el equipo de enfermería de un servicio de. JOURNAL HEALTH NPEPS. 2019; 4(2): 357-369.

16. SILVA RS, et al. Estresse ocupacional dos enfermeiros de urgência e emergência de um hospital público de Teresina (PI). 2010.

17. SILVEIRA MM, et al. Estressores e coping: enfermeiros de uma unidade de emergência hospitalar. Revista eletrônica de enfermagem. 2009; 11(4): 894-903.

18. SOUZA MT, et al. Revisão integrativa: o que é e como fazer. Einstein. 2010; 8(1) Pt 1: 102-6.

19. SOUZA SPF, et al. Preditores da Síndrome de Burnout em enfermeiros de serviços de urgência pré-hospitalar. Acta Paulista de enfermagem. 2012; 25(1): 68-73.

20. TRETTENE AS, et al. Estresse em profissionais de enfermagem atendentes em unidade de pronto atendimento. Bol. Acad. Paulista de Psicologia, São Paulo, 2016. 